\title{
Correlation Effects of Two Interacting Particles in a Circular Billiard
}

\author{
L. A. Toporowicz ${ }^{a}$ and M. W. Beims ${ }^{a, b}$ \\ a Departamento de Física, Universidade Federal do Paraná, 81531-990, Curitiba, \\ Brazil \\ ${ }^{\mathrm{b}}$ Max Planck Institut for the Physics of Complex Systems, Nöthnitzer Strasse 38, \\ 01187 Dresden, Germany
}

\begin{abstract}
The maximal Lyapunov exponent is determined numerically for two classical unequalmass interacting particles in a circular billiard. A Yukawa potential is used for the interaction between the particles. Transitions from short to long interaction ranges and from equal to infinite masses ratio between particles are discussed. Correlations effects between particles strongly determine the dynamics inside the billiard. A qualitative change in the Lyapunov exponent dependence on the interaction range between particles, is observed by the transition from weak to strong couplings. Poincaré Surfaces of Section are also used to describe the dynamics in the limit of infinite masses ratio.
\end{abstract}

Key words: Two-Particles Systems, Soft Interactions, Lyapunov exponents PACS: 05.45.-a, 05.45.Pq, 45.50.Jf

Interacting particles inside circular billiards can be used to model electrons in quantum walls. Such quantum devices are atomic-like few electron systems which may arise when homogeneous two-dimensional electron gases of heterojunctions are laterally confined to diameters comparable to the effective Bohr radius of the host semiconductor [1]. Electrons are confined inside a disk and are affected by the surrounding material which composes the semiconductor. In fact, the composition of the surrounding material may firstly, destroy the long Coulomb repulsion between electrons and secondly, change the effective mass between particles [2]. The influence of both effects on quantum energy levels and/or electrons dynamics it not obvious. However, they can be studied in a physical model by including a parameter which controls the interaction range between particles (electrons) and by allowing variations of particles masses ratio. Some of the above mentioned effects have been analysed in a quantum

Preprint submitted to Elsevier Preprint 23 December 2005 
model of two interacting (Yukawa force) particles inside a circular billiard subjected to an external magnetic field [3]. However, sometimes it is very useful to understand the classical dynamics of the correspondent quantum problem.

In this contribution we analyse the classical dynamics of two unequal-mass interacting particles as the masses ratio, the interaction range between particles and, the coupling strengths are changed. Besides the Yukawa interaction force, particles are under the action of a weak constant magnetic field and move inside a circular billiard with an infinite wall. The dynamics is analysed for three kinds of transitions: a) from long to short interaction ranges between particles, b) from equal to infinite masses ratio and c) from low to high energies, i. e. , from strong to weak couplings, respectively. All transitions are from interest because they reflect the effect of the surrounding material from the semiconductor on the physical properties and particles dynamics inside the billiard. Maximal Lyapunov exponents and Poincaré Surface of Sections are used to describe the above mentioned transitions.

The problem Hamiltonian with the weak magnetic field perpendicular to the billiard in polar coordinates is given by

$$
\begin{aligned}
& H=H_{0}^{(1)}+H_{0}^{(2)}+V\left(r_{12}\right)=E, \quad V\left(r_{12}\right)=V_{0} \frac{e^{-\alpha r_{12}}}{r_{12}}, \\
& H_{0}^{(j)}=\frac{\mathbf{p}_{j}^{2}}{2 \mu_{j}}+U\left(\left|\vec{r}_{j}\right|-r_{0}\right)+\frac{B L_{z}^{(j)}}{2 \mu_{j}} .
\end{aligned}
$$

Here, $r_{12}=\left|\vec{r}_{1}-\vec{r}_{2}\right|=\sqrt{r_{1}^{2}+r_{2}^{2}-2 r_{1} r_{2} \cos \left(\theta_{1}-\theta_{2}\right)}$ is the relative distance between the particles, with $\mu_{j}$ and $L_{z}^{(j)}$ being, respectively, the particle $j$ mass and the $z$-component of the angular momentum. $E$ is the total energy, $B$ is the intensity of the external magnetic field and $U\left(\left|\vec{r}_{j}\right|-r_{0}\right)$ represents the infinite repulsive potential of the circular billiard on the $j$-particle located at $\left|\vec{r}_{j}\right|=r_{0}$. At the boundary $r=r_{0}$, particles collide elastically with the infinite wall. The interaction Yukawa potential $V\left(r_{12}\right)$ has strength $V_{0}$ and range action $\alpha$. Values of $\alpha$ between 0 and 1, model the long range interaction and values of $\alpha \geq 6$, the short range interaction. We also consider the strong coupling limit when $E / V_{0} \sim 1$, where the interaction energy between particles is comparable to the particles kinetic energy. The weak coupling limit occurs for $E / V_{0}>>1$, where the interaction intensity between particles is much lower compared to the particles kinetic energy.

Calculation of the Maximal Lyapunov Exponent (MLE) can be done by using Benettin's algorithm [4]. Basically it assumes that little perturbations in the initial conditions will, for long times, stretch mainly along the most unstable direction in phase space. In this way, the maximal (or the largest) Lyapunov 
exponent is calculated from $\lambda_{\max }=\frac{1}{n \tau} \sum_{i=1}^{n} \ln \frac{\left|d_{i}\right|}{|d|}$, where $\frac{\left|d_{i}\right|}{|d|}$ is the stretching factor due an initial perturbation $|d|$. After each time $\tau$, the quantity $\ln \frac{\left|d_{i}\right|}{|d|}$ is evaluated and $\lambda_{\max }$ is an average over $n$ such evaluations. If $\lambda_{\max }$ converges to a positive value, the trajectory is chaotic. For all simulations done in this paper we used $\tau=0.1$ and $n=10^{5}$ for 100 initial conditions.

In order to present results clearly, we start with the most simple of the cases considered here. It is the case of infinite masses ratio $\left(\gamma=\mu_{2} / \mu_{1} \rightarrow \infty\right)$, where particle 2 has an infinite mass and is assumed to be kept fixed at a distance $R$ from the origin of the billiard. Particle 1 will move under the Yukawa repulsion due to particle 2. This situation is very similar to the Annular billiard [5], where the inner circle (an infinite wall) induces a chaotic motion as long it is not located at the origin of the billiard $(R=0.0)$. Here, as in the Annular billiard, when particle 2 is not at the origin, the motion of particle 1 is expected to get more and more chaotic as $R$ increases. This is shown in Fig. 1a) for
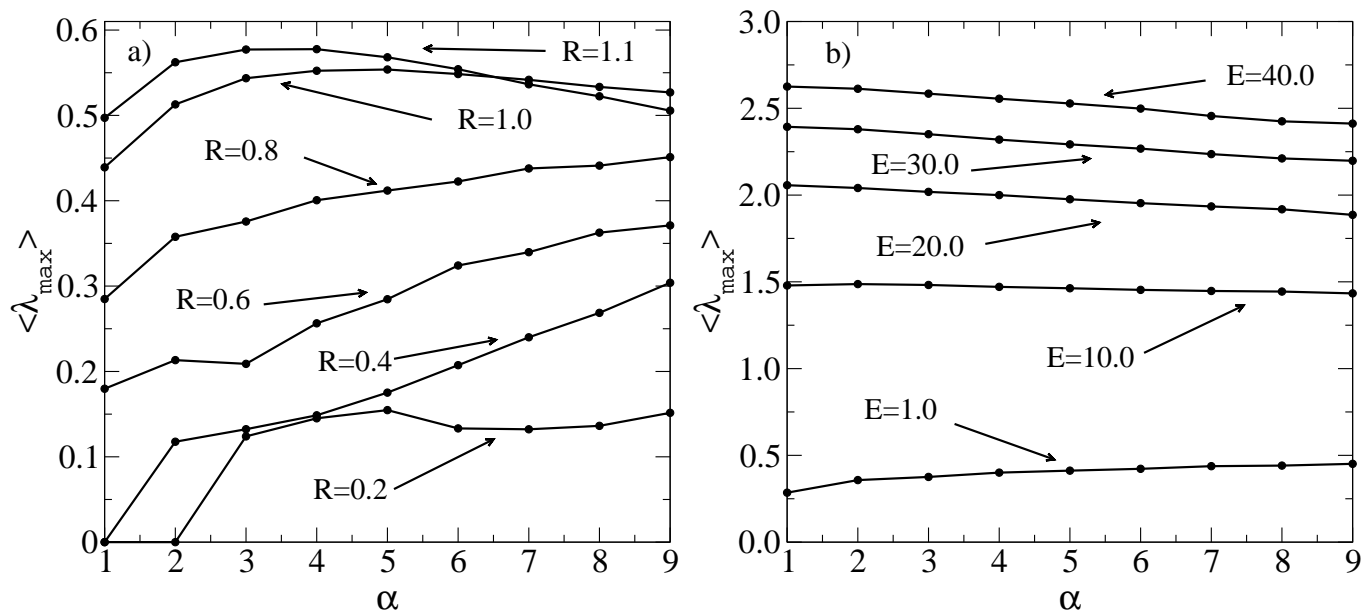

Fig. 1. Average MLE as a function of the interaction range $\alpha$ in the limit $\gamma \rightarrow \infty$, for a) $E=1.0$ and different values of $R$ and b) $R=0.8$ and different values of the energy $E$. In both cases we used $V_{0}=1.0$ and $B=0.1$.

$E=V_{0}=1.0$ and $B=0.1$. The average MLE is plotted as a function of the interaction range $\alpha$, and for different values of $R$. The average MLE increases when $R$ and $\alpha$ increase (except for the case $R=1.1$ ). The system is therefore getting more chaotic when $R$ and $\alpha$ increase. Results depend strongly on the initial position $R$ of particle 2 . The case $R=1.1$ assumes that the fixed particle 2 is located outside the billiard. In this case particle 1 is alone inside the billiard, but the finite interaction with particle 2 induces the chaotic motion. This means that particles located in the material surrounding the billiard may induce a chaotic motion inside the billiard.

Figure 1b) shows the interaction range dependence of the MLE for different values of the energy $E$. In this case $R=0.8, V_{0}=1$ and $B=0.1$. For $E=1.0$, the curve from Fig. 1a) is just repeated. For high energies we observe 
a qualitative change in the dependence of the MLE on the parameter $\alpha$. For $E=30$, for example, the MLE decreases when the short interaction limit is approached. In other words, for high energies the dynamics is more regular in the short interaction limit, exactly the opposite of what happened in the low energy regime. Since correlation effects are strong for low energies, and weak for high energies, they are responsible for the above qualitative change in the MLE behavior.

Results above can be understood better by looking at the dynamics of particle 1. Figure 2 shows the Poincaré Surface of Sections (PSS) when $V_{0}=1.0, B=$ $0.1, R=0.2$ for: a)-c) $E=1.0$ and d)-f) $E=30.0$. Plotted in this figure is $y=\sin \chi$ against $x=\theta$, where $\chi$ is the angle of the particles momentum to the collision normal of the billiard at the collision point. The angle of the collision point to the origin is $\theta[6]$. We observe that for low energies $(E=1.0)$ and low values of $\alpha$, Fig. 2a) shows mainly a regular motion. As the short interaction range limit is approached $(\alpha=6.0)$, the motion turns to be chaotic [see Fig. 2c)]. Each time the points in the PSS have values of $\sin \chi$ close \pm 1 ,
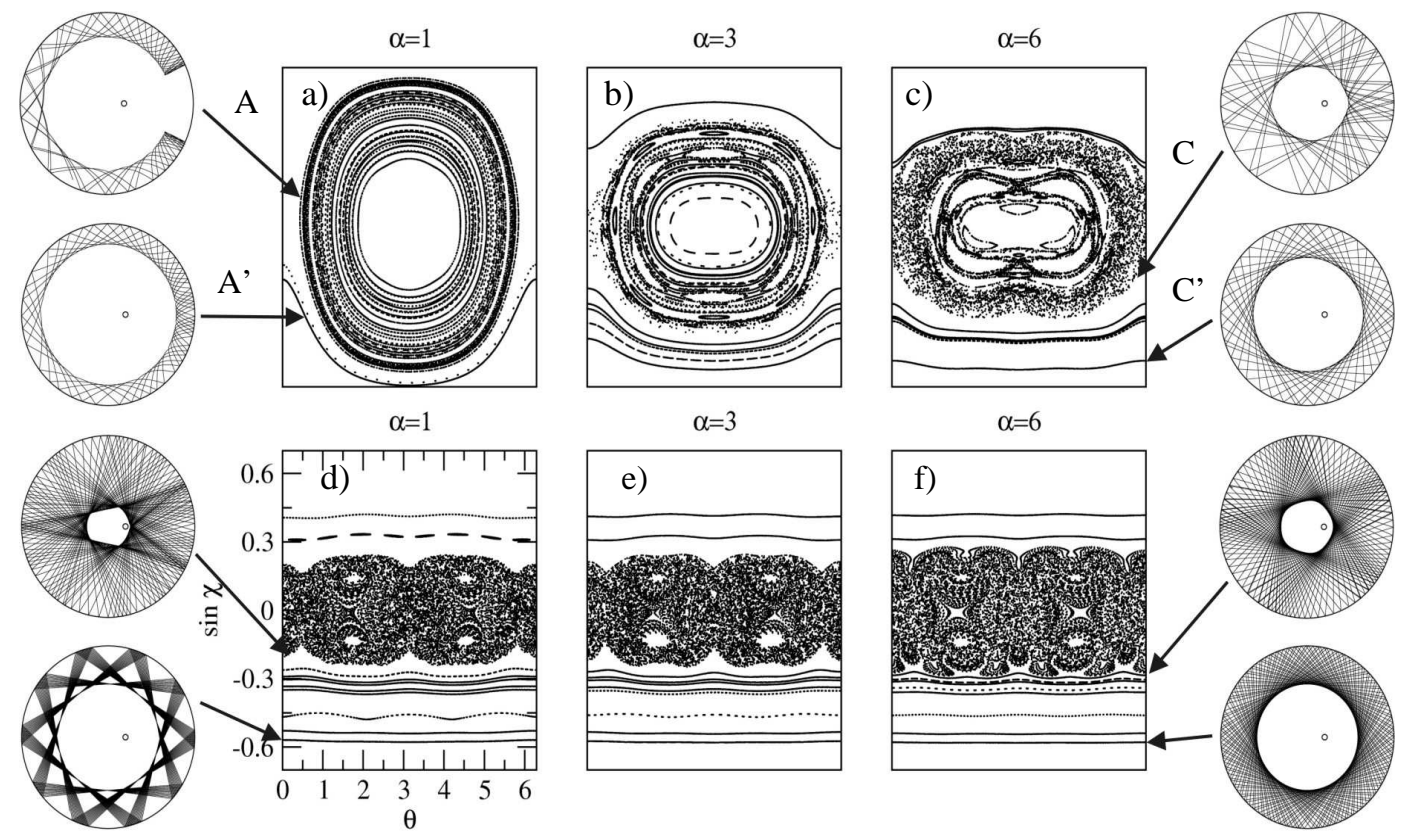

Fig. 2. Poincaré Surfaces of Sections for different values of the interaction range and for: a)-c) $E=1.0$ and d)-f) $E=30.0$. For both cases $V_{0}=1.0$ and $B=0.1$. Some example of trajectories are shown outside the PSS. Arrows indicate the respective curve in the PSS. The little circle inside the trajectories shows the position of particle 2 at $R=0.2$.

means that particle 1 collides mostly perpendicularly to the collision normal. For many initial conditions in Fig. 2a), particle 1 never collides with the wall for $\theta$ close to $2 \pi$. In other words, these trajectories never pass "behind" (to the right of) particle 2 due to the repulsive Yukawa force. This is shown by an example of one trajectory from Fig. 2a) (see arrow A). However, if an initial 
condition is started with $\sin \chi \sim-0.7$ (see arrow $A^{\prime}$ ), then the trajectory may pass on the right side of particle 2. This trajectory has the tendency to collide more perpendicularly to the collision normal when it is far from (on the left of) particle 2, and more parallel to it if it is close to (on the right of) particle 2 (see these details by the trajectory related to arrow $A^{\prime}$ ). In the short interaction range limit $(\alpha=6.0)$, where the motion is more chaotic [see Fig. 2c)], irregular orbits are those with $\sin \chi$ closer to zero, and are moving closer to particle 2. Regular orbits are moving far from particle 2 and with $\sin \chi \sim-0.6$. Examples of such trajectories are shown beside Fig. 2c) (see arrows $C$ and $C^{\prime}$ ).

Figures 2d)-f) show results for the high energies regime. In this regime, the qualitative dependence of the MLE, as a function of $\alpha$, changes if compared with results from low energies [see again Fig. 1b)]. For high energies the MLE in the short interaction limit is lower compared to the long interaction case. As a consequence, the dynamics should also be more chaotic in the long interaction range. Since both cases are chaotic, this difference is not easy to observe in the PSS. Now we consider the full Hamiltonian (1), where both particles can
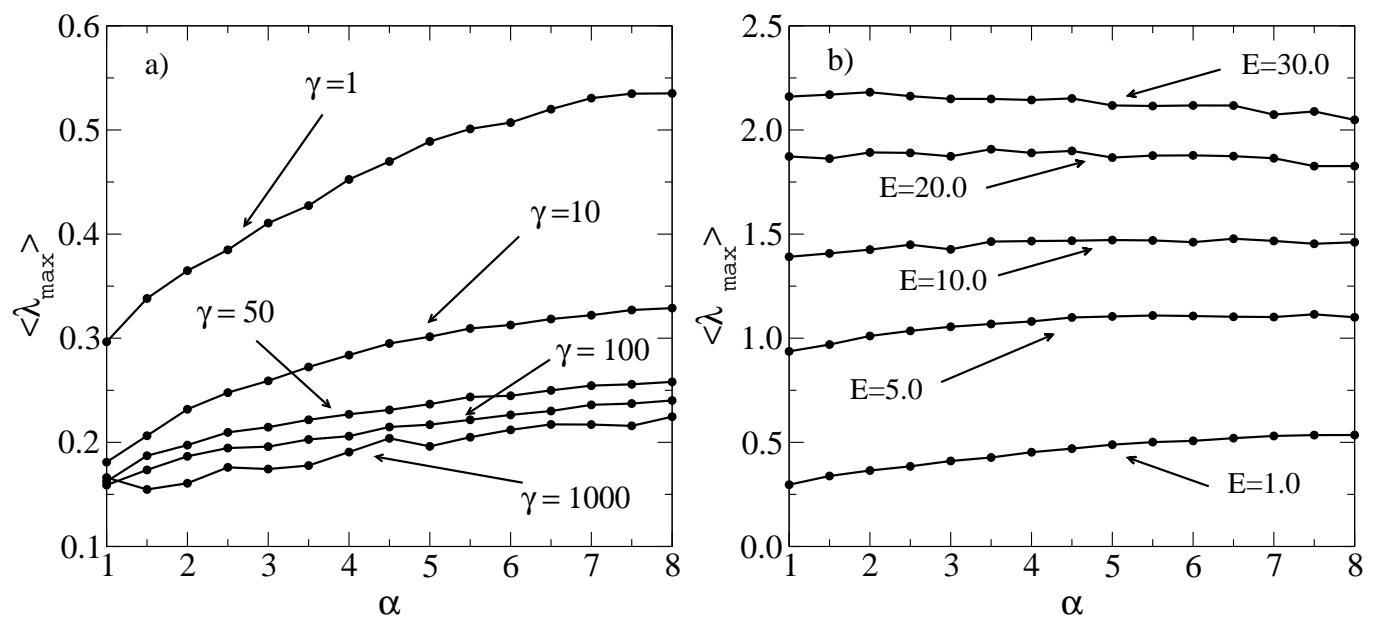

Fig. 3. Average MLE as a function of the interaction range $\alpha$ for different values of a) masses ratio $\gamma$ for $E=1.0$, and b) total energy $E$ for $\gamma=1.0$. In these cases $V_{0}=1.0$ and $B=0.1$.

move interacting which each other and with the billiard. In this case it is not possible to construct an adequate PPS which allows us to look at the dynamics of both particles. Figure 3a) shows the average MLE as function of the interaction range parameter $\alpha$ and for different values of the masses ratio $\gamma$. In this case $V_{0}=1.0, E=1.0$ and $B=0.1$. First observation is that the MLE increases as $\alpha$ increases. This means that the dynamics is getting more and more chaotic in the limit of short interaction ranges. As the masses ratio $\gamma$ increases, the MLE decreases, and the dynamics is more regular. However, the $\alpha$ dependence of the MLE remains the same. Figure 1b) shows the MLE as a function of the interaction range, but for different values of the total 
energy $E$. In this case $\gamma=1.0$. The interaction range dependence is changing qualitatively as the energy is increased. For higher energies $\left(E / V_{0}=30\right)$, the Lyapunov exponent decreases by the transition from long to short interaction ranges and the dynamics is getting more regular. This is exactly the opposite of what happened in the low energy regime.

To conclude, we observe that two particles correlations effects strongly determine the dynamics inside a circular billiard. Particles are also subjected to an external static magnetic field. The correlation between particles is responsible for a qualitative change in the interaction range dependency of the maximal Lyapunov exponent. In the limit of strong (weak) couplings, where the interaction intensity is comparable to (lower then) the particles kinetic energy [i. e. , $E / V_{0} \sim 1\left(E / V_{0}>>1\right)$ ], the dynamics is more chaotic (regular) when particles interaction range decreases. The transition from equal $(\gamma=1)$ to infinite $(\gamma=\infty)$ masses ratio between particles was also analysed. As the masses ratio increases, the MLE decreases (but not to zero). Its value will depend strongly on initial conditions of the heavy particle. This was also confirmed by analysing the dynamics in the Poincaré Surfaces of Section in the limit $\gamma=\infty$, where the heavy particle is kept fixed in space. The qualitative change of the MLE due correlation effects, as mentioned above for $\gamma=1$, was also observed in the limit $\gamma \rightarrow \infty$.

\section{Acknowledgement}

M. W. Beims thanks JM Rost and AS de Wijn for helpfull discussions and CNPq and F. Araucária for financial support.

\section{References}

[1] M. A. Reed and W. P. Kirk, Nanostructure and Fabrication, Academic, Boston, 1989.

[2] O. Gunawan, Y. P. Shkolnikov, E. P. De Poortere, E. Tutuc and M. Shayegan, Phys. Rev. Lett. 93 (2004) 246603-1; U. Merkt, J. Huser and M. Wagner, Phys. Rev. B 43 (1991) 7320.

[3] E. P. S. Xavier, M. C. Santos, L. G. G. V. Dias da Silva, M. G. E. da Luz and M. W. Beims, Physica A 342 (2004) 377.

[4] G. Benettin, L. Galgani and J-M. Strelcyn, Phys. Rev. A 14 (1976) 2338.

[5] O. Bohigas, D. Boosé, R. Egydio de Carvalho, V. Marvulle, Nucl. Phys. A 560 (1993) 197.

[6] M. Hentschel and K. Richter, Phys. Rev. E 66 (2002), 056207. 